\title{
MASTER INVESTIGATION OF ANTARES AND ICECUBE ALERTS
}

\author{
O. A. Gress ${ }^{1,2}$, V. M. Lipunov ${ }^{1,3}$, D. Dornic ${ }^{4}$, E. S. Gorbovskoy ${ }^{1}$, V. G. Kornilov ${ }^{1,3}$, N. V. Tyurina ${ }^{1}$, \\ P. V. Balanutsa ${ }^{1}$, A. S. Kuznetsov ${ }^{1}$, V. V. Vladimirov ${ }^{1}$, and D. A. Kuvshinov ${ }^{1}$
}

\begin{abstract}
This paper presents the first investigation results of possible coincidences of optical transients in the MASTER global robotic telescope net database with the localization regions of VHE and UHE neutrino alert events from ANTARES and IceCube observatories. MASTER is the first robotic telescopic system in Russia, dedicated to optical observation and registration of the most energetic and fastest explosive processes in the Universe. The goal is a deeper and more thorough study of space and time coincidences of the MASTER optical transients and high energy neutrinos.
\end{abstract}

\section{RESUMEN}

Este artículo presenta los primeros resultados de investigación de la posible coincidencias de fuentes transitorias ópticas en la base de datos de la red de telescopios robóticos MASTER con la localización de eventos de alerta de neutrinos VHE y UHE por los observatorios ANTARES y IceCube. MASTER es el primer sistema telescópico robótico en Rusia, dedicado a la observación óptica y registro de los eventos más energéticos y los procesos explosivos más rápidos en el Universo. El objetivo es un estudio más profundo del espacio a partir de las coincidencias temporales de los transitorios ópticos detectados por MASTER y los neutrinos de alta energía.

Key Words: telescopes — neutrinos

\section{INTRODUCTION}

The origin of high-energy cosmic rays is still a mystery. It is expected that the cosmic rays and neutrinos are produced in the hadro-nuclear and photo-hadronic interactions with ambient matter. Transient objects are interesting because they correspond to a short-time release of a large amount of energy. In principle, the neutrino data and electromagnetic measurements investigation by a multimessenger approach (Santander 2016; Smith et al. 2013) can increase our ability for understanding of the neutrino sources and help to solve long-standing problems in astrophysics such as the origin of cosmic rays.

This article presents the investigation results of possible coincidences of optical transients (OTs) in the MASTER global robotic telescope net (Lipunov et al. 2010) database with the localization regions of Very High Energy (VHE) and Ultra High Energy

\footnotetext{
${ }^{1}$ M. V. Lomonosov Moscow State University, SAI, Universitetsky pr., 13, 119234, Moscow, Russia (lipunov2007@gmail.com).

${ }^{2}$ Applied Physics Institute, Irkutsk State University, Gagarin blvd, 20, 664003, Irkutsk, Russia (mr.grol08@mail.ru).

${ }^{3}$ M.V.Lomonosov Moscow State University, Physics Department, Vorobievy hills, 1, 119991, Moscow, Russia.

${ }^{4}$ Aix Marseille University, CNRS/IN2P3, CPPM UMR 7346, 13288, Marseille, France.
}

(UHE) neutrino alert events from ANTARES and IceCube observatories.

\section{NEUTRINO OBSERVATORIES}

The primary goal of the neutrino detectors (Spiering 2017) is identifying the sources of highenergy cosmic rays. Atmospheric neutrinos are the main source of background in the search for astrophysical neutrinos.

The ANTARES neutrino telescope (Ageron et al. 2011) has been continuously operating since 2008. Located deep $(2500 \mathrm{~m})$ in the Mediterranean Sea, $40 \mathrm{~km}$ from Toulon (France), it is a 10 megaton-scale underwater Cherenkov array of photosensors, detecting neutrinos with energies $\geq 0.1 \mathrm{TeV}$. The median angular errors of neutrinos detected by ANTARES are $\sim 0.4^{\circ}$ at $10 \mathrm{TeV}$ and $\sim 0.7^{\circ}$ at $1 \mathrm{TeV}$. These values correspond to the radius of a gaussian error box which contains $50 \%$ of the events. The value of $1.4^{\circ}$ is a conservative value $\sim 3 \sigma$. ANTARES alert system is operational since early 2009 (Ageron et al. 2012).

IceCube is a $1 \mathrm{~km}^{3}$-size neutrino detector (Aartsen et al. 2017a) installed in the ice at the geographic South Pole in Antarctica between depths of $1450 \mathrm{~m}$ and $2450 \mathrm{~m}$ and in operating since 2010 . A real-time event selection for single VHE track events with high probability of being of astrophysical origin (Smith et 


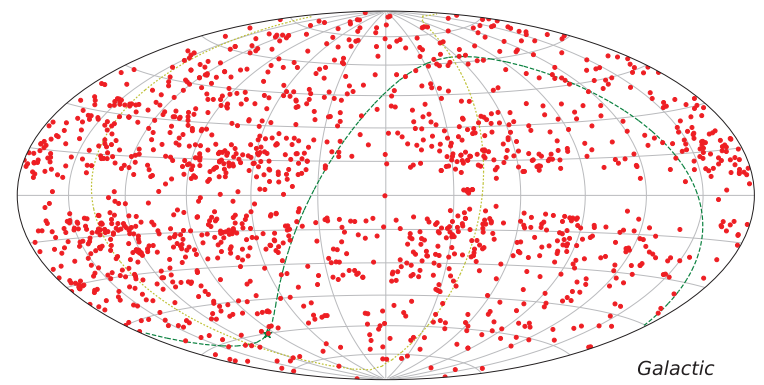

Fig. 1. MASTER optical transients skymap (Galactic coordinates). In the period since April 2005 to September 2017 the MASTER robotic telescope network discovered 1470 optical transients covering different astrophysical types. The red dots are OTs, the green dashed line indicates the celestial equator and the light-brown dot-dashed line indicates the supergalactic plane.

al. 2013) and has been operating since of 2016. IceCube has detected the first astrophysical VHE neutrinos.

\section{MASTER-NET AUTO-DETECTION SYSTEM}

MASTER (Mobile Astronomy System of Telescope Robots) global robotic telescope net (Lipunov et al. 2010) was developed to discover prompt optical emission from gamma-ray bursts (Troja et al. 2017), to investigate LIGO/Virgo large error fields to discover optical counterparts of gravitational wave sources (Lipunov et al. 2017a,b), to investigate IceCube and ANTARES error-boxes in search of possible counterparts of high enrgy neutrino alerts, and to produce a fast sky survey to find optical transients down to a limiting magnitude of $19^{m}-20^{m}$.

MASTER global robotic net is the first robotic telescope system in Russia, began to be created through the efforts of scientists from Lomonosov Moscow State University (SAI) and the Moscow "Optika" Association in 2002. Now there are Lomonosov MSU, Blagoveschensk State Pedagogical Institute, Irkutsk State University, Institute of Astrophyics of Canarias Islands, South African Astronomical Observatory, San Juan National University (Astronomical Observatory of Felix Aguilar OAFA) collaboration, involved in MASTER.

MASTER global robotic net consists of 8 observatories, located in Russia (MASTER-Amur, -Tunka, -Ural, -Kislovodk, -Tavrida), in Spain (MASTER-IAC), in South Africa (MASTERSAAO) and in Argentina (MASTER-OAFA), equipped with identical optical instruments: (1) wide-field channel of twin 40-cm telescopes with own designed photometer ${ }^{5}$ (Lipunov et al.

\footnotetext{
${ }^{5}$ it contains two W (white) filters, BVRI filters of Johnson-
}

2010; Kornilov et al. 2012; Gorbovskoy et al. 2010; Lipunov et al. 2016a), designed to study the prompt (synchronous multi-colour and polarimetric observations) optical emission of gamma-ray bursts, search for supernovae, follow-up of gravitational waves and neutrino alerts, and (2) very wide field channel with field of view (FOV) of $2 \times 384 \mathrm{deg}^{2}$ for detecting objects down to $12^{m}$ per 5 sec exposition.

The key MASTER feature is its own autodetection real-time ( $1-2$ min after CCD readout) software. With this auto-detection system MASTER has discovered 1470 OTs during since 2005 April to 2017 September covering the different astrophysical types: kilonova MASTER OTJ130948.10232253.3/SSS17a, optical GRB counterparts (including prompt emission), supernovae (SN), novae, dwarf novae (WD), cataclysmic variables, flares of Actice Galactic Nuclei (AGN) and quasars (QSO), as well as anti-transients, comets, potentially hazardous asteroids, short flashes of UV Cet type and very short OTs of unknown nature.

Figure 1 shows the sky map in galactic coordinates of MASTER optical transients. It should be noted that the flow of discoveries is quite intense and, as a result, only $10 \%$ of the discovered MASTER transients was subjected to more or less detailed investigation of their nature.

\section{OTs SEARCH ALGORITHM}

\subsection{Survey Mode by Neutrino Alerts}

MASTER receives ANTARES and IceCube alerts by socket (usually) within 20 sec. Depending on the neutrino alert coordinates, time and meteorological conditions, all telescopes go automatically to the alert or inspection mode into the sky region from which the neutrino event came. The neutrino alert can be observed with 1 up to 7 telescopes. Observations last from a few minutes to an hour every subsequent night for up to 2 months after the alert time. The total time of observation of each alert is several hours in the field of view of 8 square degrees.

\subsection{Technique of Searching for OTs by Neutrino Alerts in the MASTER Database}

MASTER OTs detection software (Lipunov et al. 2010) automatically identifies all optical sources at every image, marking separately known and new objects (including mooving ones), performing calibration and photometry at 4 square degrees in real time (1-2 min after CCD readout for all procedures). With the exception of a few cases (Lipunov et al.

Cousins photometric system and two linear polarizers 


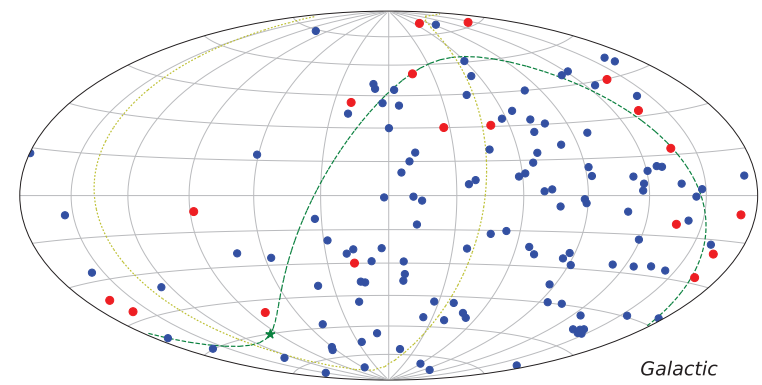

Fig. 2. Skymap of arrival directions of neutrino events (Galactic coordinates). The blue circles are ANTARES alerts, the red circles are IceCube alerts.

2016b; Aartsen et al. 2017b) there was not obvious relationship between the optical counterpart and the single VHE neutrino. Therefore, there was a need for a deeper and more careful study of the spatialtemporal correlations of such events during all OTs registration time periods.

The technique of searching for OTs is as follows: (1) OTs candidates examination, discovered or supposed to be discovered, (2) careful study of each candidate using the analysis of astronomical frames, (3) searching for additional information in astronomical catalogues, and (4) recording the received information in a single format for further analysis.

The following criteria were applied in the search for OT processes by: (1) localization area: $\sim 2$ ANTARES error-box and $\sim 1$ IceCube error-box, (2) time (before and after alert time), and (3) event spatial coincidences.

The analysis of possible neutrino sources is also carried out on diffrent optical transient types.

\section{BRIEF STATISTICS OF SEARCH RESULTS}

\subsection{Skymap of Arrival Directions of Neutrino Events}

During the period since 2015 Jan to 2017 Sep, we obtained 124 ANTARES neutrino alerts. This gives us an average ANTARES alert counting rate is of $\sim 1$ event per 6 days.

During the period since 2016 Apr to 2017 Sep, we obtained 18 IceCube neutrino alerts. The average IceCube alert counting rate is of $\sim 1$ event per month.

The sky map in galactic coordinates of the arrival direction of VHE and UHE neutrino alerts recorded by ANTARES and IceCube observatories is shown at Figure 2.

\subsection{ANTARES Alerts}

The neutrino events time intervals spectrum $N(\Delta t)$ is rather well fitted by the exponential depen-
TABLE 1

ANTARES $\nu$ ALERTS AND ASSOCIATED OTs
INSIDE ERROR-RADIUS OF $1.4^{\circ}$

\begin{tabular}{lrr}
\hline Year & $\nu$-alerts & \\
\hline 2015 Jan-Dec & 40 & OTs $\left(\leq 1.4^{\circ}\right)$ \\
2016 Jan-Dec & 50 & 13 \\
2017 Jan-Sep & 34 & 8 \\
\hline Total b $^{\text {b }}$ & 124 & 26 \\
\hline
\end{tabular}

${ }^{a}$ Number of neutrino alerts for a given time, which were detected and used in search for associated OTs.

${ }^{\mathrm{b}}$ Among 124 alerts it is only 22 neutrino events coincide with OTs inside sky region with radius of $1.4^{\circ}$.

TABLE 2

ICECUBE $\nu$ ALERTS AND ASSOCIATED OTs INSIDE ERROR-BOX

\begin{tabular}{lrr}
\hline Year & $\nu$-alerts & \multicolumn{1}{c}{ OTs } \\
\hline 2016 Apr-Dec & 9 & 5 \\
2017 Jan-Sep & 9 & 7 \\
\hline Total & 18 & 12 \\
\hline
\end{tabular}

${ }^{a}$ Among these IceCube VHE astrophysical neutrinos, there are four events (IceCube-160813, -160827, -160919 and -170503$)$ with a very big error-box $>1.4^{\circ}$.

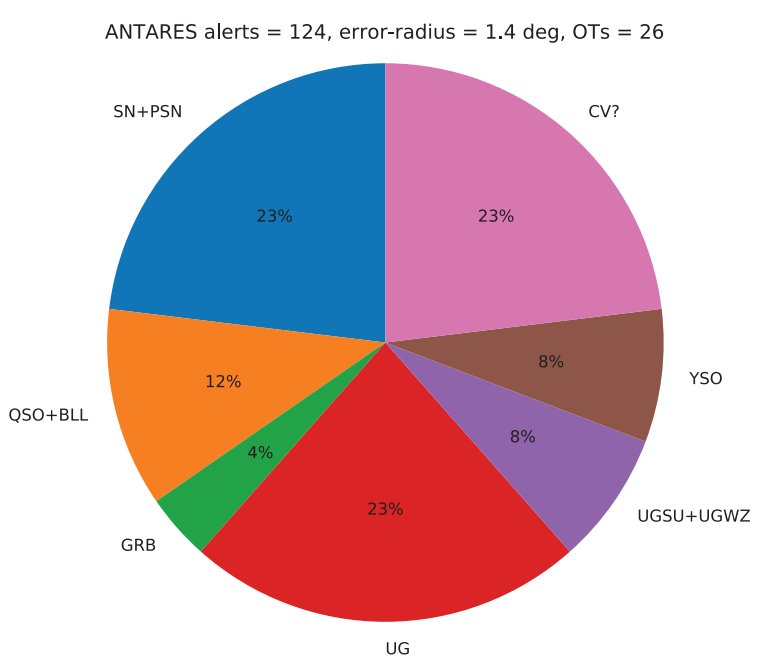

Fig. 3. Distribution by OTs types. OT positions coincide with the ANTARES high-energy neutrino arrival directions in the period since 2015 Jan to 2017 Sep within the error-radius of $1.4^{\circ}$. Note: GRB is $4 \%$ and corresponds 1 possible event. 
TABLE 3

ANTARES NEUTRINO ALERTS AND OPTICAL TRANSIENTS ${ }^{a}$

\begin{tabular}{|c|c|c|c|c|c|c|}
\hline Alert Name & $Z,^{\circ}$ & OT Name ${ }^{\mathrm{b}}$ & Type & $W$, mag & $\Delta_{O T},{ }^{\circ}$ & $\Delta t$, month \\
\hline AA-150321B & -39.7 & CRTS CSS131224 & SN.II & 17.9 & 0.713 & -14.5 \\
\hline AA-150607 & -00.5 & MASTER OTJ005720.90 & QSO & 19.1 & 1.385 & -07.0 \\
\hline AA-151020A & -71.5 & GRB 161023A & GRB & 12.7 & 0.376 & +12.0 \\
\hline AA-151213 & -82.2 & MASTER OTJ073936.05 & UG & 18.2 & 0.738 & +09.0 \\
\hline AA-151213 & -82.2 & GSC2.3 S620082622 & CV? & 17.4 & 0.756 & +15.0 \\
\hline AA-160101 & -21.2 & MASTER OTJ063100.30 & UG & 14.9 & 1.259 & -27.0 \\
\hline AA-160111 & -56.8 & 3FGL J2345.2-1554 & BLL & 15.1 & 0.616 & -01.5 \\
\hline AA-160120 & -27.3 & SVM J063133.22-652057.0 & YSO & 19.7 & 1.120 & +0.17 \\
\hline AA-160120 & -27.3 & $06^{h} 37^{\prime} 04.9^{\prime \prime}-65^{\circ} 47^{\prime} 46.7^{\prime \prime}$ & CV? & 18.5 & 1.075 & +10.5 \\
\hline AA-160309A & -16.2 & PNV J03093063+2638031 & UGWZ & 11.6 & 0.619 & -16.0 \\
\hline AA-160309A & -16.2 & PS15dpq & SN.Ia & 17.9 & 0.895 & -02.5 \\
\hline AA-160319 & -39.8 & MASTER OTJ225624.49 & PSN & 18.4 & 1.134 & -09.5 \\
\hline AA-160415A & -10.2 & ASASSN-14mn & UG & 15.4 & 1.220 & +0.02 \\
\hline AA- 160617 & -04.3 & $19^{h} 47^{\prime} 35.7^{\prime \prime}-24^{\circ} 46^{\prime} 50.9^{\prime \prime}$ & CV? & 18.5 & 1.386 & +01.0 \\
\hline AA-160902 & -08.8 & CSS 080208:103317+072119 & UGSU & 13.9 & 1.444 & +01.5 \\
\hline AA-160929 & -74.8 & MASTER OTJ055250.71 & UG & 17.9 & 1.326 & -11.5 \\
\hline AA-161031 & -11.9 & SDSS J052549.10+040530.7 & CV? & 18.3 & 0.977 & +0.37 \\
\hline AA-161225 & -76.9 & MASTER OTJ040938.68 & PSN & 17.3 & 0.848 & -15.5 \\
\hline AA-170202 & -78.8 & GSC2.3 S5LU013545 & CV? & 19.3 & 1.442 & +0.70 \\
\hline AA-170202 & -78.8 & MASTER OTJ105824.56 & UG & 16.7 & 1.024 & +02.5 \\
\hline AA-170301 & -29.2 & SN $2016 \mathrm{csr}$ & SN.Ia & 17.3 & 0.709 & -09.0 \\
\hline AA-170317 & -77.2 & SVM J091029.18-503203.8 & YSO & 16.6 & 0.497 & -01.0 \\
\hline AA-170512 & -59.5 & MASTER OTJ122201.31 & $\mathrm{UG}$ & 18.8 & 1.374 & -13.0 \\
\hline AA-170531 & -12.5 & MASTER OTJ054404.79 & PSN & 17.8 & 1.370 & -19.5 \\
\hline AA-170717 & -55.4 & GSC2.3 SC7N010673 & CV? & 16.7 & 0.992 & -25.5 \\
\hline AA-170903 & -31.1 & SDSS J104304.75+020549.1 & QSO & 18.5 & 1.047 & -06.0 \\
\hline
\end{tabular}

${ }^{\mathrm{a}} \mathrm{AA}$ - ANTARES alert, each neutrino error-box is $1^{\circ} ; Z$ - zenith angle of neutrino event in the observation time, degree; $W$ - star magnitude of optical flash in white light (unfiltered); $\Delta_{O T}$ - deviation of the OT position from the neutrino arrival direction, degree; $\Delta t=t_{O T}-t_{\nu}$, i.e. the time of the transient flare relative to the alert.

${ }^{\mathrm{b}}$ Designation for OTs: CRTS CSS131224 J033239+014313, MASTER OTJ005720.90+282557.1, MASTER OTJ225624.49-333456.0, MASTER OTJ055250.71-395431.6, MASTER OTJ040938.68-541316.9, MASTER OTJ105824.56-351922.9, MASTER OTJ122201.31-271527.5, MASTER OTJ054404.79-314655.2

dence of $N(\Delta t) \simeq \exp (-\Delta t / 0.16)$ (where $\Delta t-$ difference in the event times). So, a random nature of neutrino detection is confirmed very well. An excess in the angle distribution of neutrino arrivals along zenith angle is observed at angles in the ranges from $-25^{\circ}$ to $0^{\circ}$, which can be caused by atmospheric neutrinos (so as VHE neutrino trigger turned out to be atmospheric neutrinos with non-zero false alarm rate) (Ageron et al. 2012).

A brief summary of the neutrino alerts and associated OTs found in the MASTER database is given in Table 1 . Note that this detected 26 optical transients coincide with only 22 neutrino alerts for the full observation period within $1.4^{\circ}$. In the case that the search area will increase 4 times $\left(3^{\circ}\right.$ errorradius), also the number of OTs will increase to 138 (likely random coincidence will be higher). Figure 3 shows the OTs distribution from Table 1 by their types.

\subsection{IceCube Alerts}

The IceCube neutrino alert sytem (Aartsen 2015; Spiering 2017) owns the registration of the astrophysical $(\sim 6$ alerts per year) and cosmological $(\sim 5$ alerts per year) origin neutrinos at energies from $500 \mathrm{TeV}$ to $1 \mathrm{EeV}$. 
A brief summary of the IceCube neutrino alerts ${ }^{6}$ and associated OTs found in the MASTER database is given Table 2 .

\section{ANTARES NEUTRINO ALERTS: POSSIBLE TRANSIENTS}

The results of search for possible OTs in the ANTARES alert localization error-boxes within angular resolution of $1.4^{\circ}$ are given in the Table 3 . Types of transients such as SN (or PSN), UG (Dwarf Novae, i.e. U Geminorum variability type), CV? (possible dwarf nova outbursts or unknown type variability) are the most numerous (6 events in each group). In the following, we present characteristic values and dependencies for the most interesting cases of OTs that can be related to neutrinos detected by ANTARES.

- UG-type variables outbursts. There are 4 UGtype OTs with a large (several months) time interval before the neutrino alert time, and substantially outside the limits of $1^{\circ}$. MASTER OTJ073936.05 was discovered 9 days after the alert with a relatively small angular error-box of $0.74^{\circ}$. A bright $15^{m}$ flash from the known ASASSN-14mn was observed 12 hours after the alert, but AA-160415A event had a doubtful arrival direction.

We have a quite good angular coincidence of the AA-160309A alert and the very bright $12^{m}$ UGWZtype object PNV J03093063+2638031 (Munari et al. 2015) within $0.6^{\circ}$. This transient was observed 16 months before alert and moreover this alert had a doubtful arrival direction.

- Variables of unknown types. Mysterious weak CV?-type OTs also have a long times (a few months) before and after the alert times, except for two possible cases: AA-161031 and AA-170202. Almost all have positions outside the limits of $1^{\circ}$ from alerts.

- Young Stellar Object bursts. There is a rather interesting case of a bright flash of YSO SVM J091029.18-503203.8 a month before AA-170317 event within $0.5^{\circ}$.

- Gamma Ray Bursts. A single coincidence of a bright optical flash $12.7^{m}$ from a long $\gamma$-ray burst GRB 161023A (Gorbovskoy et al. 2016) and AA$151020 \mathrm{~A}$ event within of $0.38^{\circ}$ region was found. This GRB 161023A had a photometric redshift $z=$ 2.708 and a $\gamma$-ray duration $t_{90}=80 \mathrm{sec}$. GRB 161023A optical counterpart was observed $\sim 1$ year

\footnotetext{
${ }^{6}$ This information about AMON_ICECUBE_HESE and AMON_ICECUBE_EHE Events can be found in the corresponding tables on addresses: https://gcn.gsfc.nasa.gov/ amon_hese_events.html and https://gcn.gsfc.nasa.gov/ amon_ehe_events.html
}

after the neutrino alert and its optical observation time was $\sim 130 \mathrm{~min}$.

- Supernovae outbursts. We have six single neutrino events associated with SNe: PSN-3, SN Ia -2 and SN II-1. All magnitudes of the observed optical flashes from SNe (CRTS CSS131224 J033239+014313, SN PS15dpq and SN 2016csr) are $\sim 18^{m}$ and outburst times precede the alert times within a few months, in addition their deviations from the alert positions are $\sim 0.8^{\circ}$.

- Quasar bursts. Two quasars had weak $\left(19^{m}\right)$ optical flashes approximately six months before corresponding neutrino alerts with the deviations within $1^{\circ}$ from alerts..

- Optical flare of BL Lac 3FGL J2345.2-1554 up to $15^{m}$ was 1.5 month before AA-160111. Deviation of this alert position from the blazar is $0.62^{\circ}$. We can find another alert AA-150830 3 month before this blazar optical flash, but in this case the deviation is $2.38^{\circ}$.

\section{ICECUBE VHE NEUTRINO ALERTS: POSSIBLE TRANSIENTS}

The results of MASTER search for possible OTs in the region localization of IceCube VHE neutrino alerts are given in the Table 4 . It should be noted that neutrino error-boxes are $\sim 1^{\circ}$ and in most cases reach $\sim 3^{\circ}$. In the case $\sim 3^{\circ}$ error-boxes, it is difficult to talk about the OTs and neutrinos coincidence, since on large area we can find many randomly coincident active objects. All OTs have an appearance time earlier than the alert occurred and this interval has a range of several months to 2 years! In the following, we present the main dependencies for possible OTs that can be associated with IceCube astrophysical neutrinos.

- UG-type outbursts. Only two transients, i.e. Gaia16agq and MASTER OTJ145056.15, coincide within the localization accuracy $\left(\sim 1^{\circ}\right)$ and 4 and 15 months before ICA-160814 and ICA-170506 alerts respectively.

- Variables of unknown type. Despite the small value of localization region of MASTER OTJ230921.67 $\left(\sim 0.2^{\circ}\right)$ with respect to ICA-170503, this alert itself is very badly defined (error-box is $2.86^{\circ}$ ).

- Supernovae outbursts. Here we have four supernovae of different types (SN ASASSN-14ad, Ia; SN MASTER OTJ113930.78, II; SN 2014de, Ia and SN 2015ah, Ib), having common characteristic properties with respect to neutrino alerts. All of them have a visible star magnitude of $\sim 17^{m}$, very close distances, their outbursts happened around 2 years before alerts and they are inside alert error-boxes. 
TABLE 4

ICECUBE NEUTRINO ALERTS AND OPTICAL TRANSIENTS ${ }^{\mathrm{a}}$

\begin{tabular}{lllllllc}
\hline Alert Name & $Z{ }^{\circ}$ & $\Delta_{\nu},{ }^{\circ}$ & OT Name $^{\mathrm{b}}$ & Type & $W$, mag & $\Delta_{O T},{ }^{\circ}$ & $\Delta t$, month \\
\hline ICA-160813 & -18.7 & 2.79 & ASASSN-14ad & SN.Ia & 16.3 & 0.851 & -29.5 \\
ICA-160814 & +32.1 & 1.49 & Gaia16agq & UG & 18.7 & 1.074 & -04.0 \\
ICA-160827 & -25.5 & 2.90 & MASTER OTJ113930.78 & SN.II & 16.7 & 2.987 & -22.5 \\
ICA-160919 & -18.1 & 3.70 & SU Tau, RCB & VAR & 13.7 & 1.878 & +05.5 \\
ICA-161210 & -15.0 & 1.00 & SN 2014de & SN.Ia & 17.2 & 0.783 & -27.5 \\
ICA-170503 & -02.2 & 2.86 & SN 2015ah & SN.Ib & 16.3 & 2.812 & -20.0 \\
ICA-170503 & -02.2 & 2.86 & MASTER OTJ231846.26 & UV & 18.1 & 2.788 & -20.0 \\
ICA-170503 & -02.2 & 2.86 & SN 2016eky & SN.Ia & 17.8 & 2.651 & -08.0 \\
ICA-170503 & -02.2 & 2.86 & MASTER OTJ230921.67 & CV? & 18.0 & 0.154 & -08.0 \\
ICA-170503 & -02.2 & 2.86 & MASTER OTJ230153.13 & UV & 18.7 & 2.086 & -07.0 \\
ICA-170506 & +26.1 & 1.23 & MASTER OTJ145056.15 & UG & 16.3 & 1.065 & -15.0 \\
ICA-170607 & -12.8 & 1.04 & MASTER OTJ094545.44 & UG & 18.7 & 0.484 & -62.0 \\
\hline
\end{tabular}

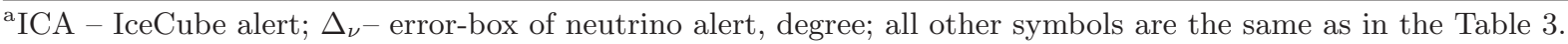

${ }^{\mathrm{b}}$ Designation for OTs: MASTER OTJ113930.78+251141.0, MASTER OTJ231846.26+044416.1, MASTER OTJ230921.67+033221.4, MASTER OTJ230153.13+021940.5, MASTER OTJ145056.15-255526.9, MASTER OTJ094545.44+125604.6.

\section{DISCUSSION}

Several source classes are considered candidates for the production of VHE neutrinos (Franckowiak 2017), where cosmic rays are produced and efficiently accelerated:

(1) star-forming galaxies, but they can not produce more than $30 \%$ (at $100 \mathrm{TeV}$ ) of the diffuse neutrino flux (Bechtol et al. 2017);

(2) blazars, but they have a weak correlation at significance level of $\sim 1 \%$ as VHE neutrino candidates (Padovani et al. 2016);

(3) GRBs, where neutrino emission will be collimated and in temporal coincidence with the prompt $\gamma$-ray emission and this contribution is $\leq 1 \%$ of the observed diffuse neutrino flux (Aartsen et al. 2016);

(4) $\mathrm{SNe}$, where we have 2 models that predict VHE neutrino production: a) SN Ic generate relativistic jets that interact with the star envelope media (Tamborra \& Ando 2016); b) SN IIn explode in a dense circum-stellar media (Zirakashvili \& Ptuskin 2016); After the ejecta interactions with dense media, neutrinos are expected to be produced on time scales of months to years.

On the other hand, so far the space and time clustering was not found in the neutrino data obtained by ANTARES and IceCube observatories (AdrianMartínez et al. 2016; Aartsen et al. 2017c). It is assumed that un-localized neutrino sources are possible, particularly those generated by the interaction of high-energy protons with relic radiation.

After the discovery of a WD/cool star binary AR Sco as white dwarf pulsar (Marsh et al. 2016; Buckley et al. 2017) it is clear that a large number of WDs can be good candidates for cosmic ray and neutrino sources. VHE neutrino can be created after pulsar relativistic jet collision with optical companion. Nevertheless WD can be invisible in this moment (Lipunov et al. 2016b).

With a large angular uncertainty of the events identification, a random coincidence of several events in some region of the sky is quite possible. We can find about $800( \pm 500)$ different star objects in the process of searching for possible candidates for neutrino sources in astronomical databases on an area within an angular resolution of $\sim 1^{\circ}$ (on average over several areas of the celestial sphere). With this approach, among other active objects we can find $\sim 50$ quasars, $\sim 10$ X-ray sources and $\sim 10$ radio sources. Every year, astronomical observatories detect $\sim 800$ supernovae brighter than $18^{m}$, or $\sim 100$ supernovae brighter than $16^{m}$ (Gal-Yam et al. 2013). Currently, on the all star sky, we have a large number of $\sim 15000$ cataclysmic variables (dwarf novae and $\mathrm{CV}$ ) and $\sim 800$ optically bright cataclysmics variables UGSU (and similar) type. 
Therefore, we can expect the following probabilities of random coincidence ${ }^{7}$ of optical transients with the neutrino alert events: CV - 20 events, UGSU 10 events, supernovae -10 events, quasars $-\sim 1$ event. In principle, the obtained result of searching for possible OTs coinciding with single neutrino events does not contradict such a simple estimation of random coincidences.

If neutrino luminosities are too low for source identification from neutrino measurements alone, we might be able to identify these sources and their origins by gamma-ray, optical, and X-ray observations in the localization areas of the individual high-energy neutrino events.

\section{CONCLUSION}

The search for transients in the MASTER database in the indentification purposes of the possible optical counterparts for VHE neutrino events from ANTARES and IceCube observatories was presented. A preliminary analysis of the obtained data on possible spatial-temporal coincidences for all survey time of MASTER telescopes is on-going. The discovered regularities in the apparent random coincidence of neutrino alerts and transients require a further study of spatial-temporal correlations and the additional information attraction in the spirit of multi-messenger astronomy.

Acknowledgments: The MASTER project is supported in part by the Development Programme of Lomonosov Moscow State University, Moscow Union OPTICA, Russian Science Foundation 16-12-00085; OG was supported by RFBR 17-52-80133.

\section{REFERENCES}

Aartsen, M. G., et al. 2015, ApJ, 811, 52

Aartsen, M. G., et al. 2016, AJ, 824, 115

Aartsen, M. G., et al. 2017a, JInst, 12, 12

Aartsen, M. G., et al. 2017b, A\&A, 607, A115

Aartsen, M. G., et al. 2017c, AJ, 835, 151

Adrian-Martínez, S., et al. 2016, AJ, 823, 65

Ageron, M., et al. 2011, NIMPA, 656, 11

Ageron, M., et al. 2012, APh, 35, 530

Bechtol, K., et al. 2017, AJ, 836, 47

Buckley, D. A. H., et al. 2017, NatAs, 1, 0029

Franckowiak, A. 2017, JPhCS, 888, 2009

Gal-Yam, A., Mazzali, P., Manulis, I., \& Bishop, D. 2013, arXiv:1103.5165v2; http://www. rochesterastronomy . org/sn2017/snstats.html

Gorbovskoy, E. S., et al. 2010, AdAst, 2010, 1

Gorbovskoy, E., et al. 2016, GCN CIRCULAR, 20102; https://gcn.gsfc.nasa.gov/other/161023A.gcn3

Kornilov V. G., et al. 2012, ExA, 33, 173

Lipunov V. M., et al. 2010, AdAst, 2010, 349171

Lipunov, V. M., et al. 2016, RMxAC, 48, 42L

Lipunov, V. M., et al. 2016, GCN CIRCULAR, 19888; https://gcn.gsfc.nasa.gov/gcn3/19888.gcn3

Lipunov, V. M., et al. 2017, ApJ, 850, 1

Lipunov, V. M., et al. 2017, MNRAS, 465, 3656

Marsh, T. R., et al. 2016, Natur, 537, 374-377

Munari, U., Jurdana-Sepic, R., Ochner, P., \& Cherini, G. 2015, A\&A, 584, A12

Padovani, P., et al. 2016, MNRAS, 457, 3582

Santander, M. 2016, arXiv:1606.09335v1

Smith, M. W. E., et al. 2013, APh, 45, 56

Spiering, C. 2017, arXiv:1711.08266v1

Tamborra, I. \& Ando, S. 2016, PhRvD, 93, 3010

Troja, E., et al. 2017, Natur, 547, 425

Zirakashvili, V. N. \& Ptuskin, V. S. 2016, APh, 78, 28

\footnotetext{
${ }^{7}$ We assume that the number of neutrino events is $\sim 100$ (close to observed) and take into account the formula: $P=\Delta / S$, where $\Delta$ - the area with a radius of $1^{\circ}, S$ - the celestial sphere total area is $41253 \mathrm{deg}^{2}$.
} 